\title{
AUTOFocus: Reaching a target in the prostate with a 3D-ultrasound image-based control law
}

\author{
R. Chalard ${ }^{1}$, D. Reversat ${ }^{1}$, G. Morel $^{1}$, and M.A. Vitrani ${ }^{1}$ \\ ${ }^{1}$ Sorbonne Université, CNRS UMR 7222, INSERM U1150, Institut des Systèmes \\ Intelligents et Robotique (ISIR), F-75005, Paris, France \\ \{chalard,reversat,morel,vitrani\}@isir.upmc.fr
}

\section{INTRODUCTION}

Biopsies are the clinical standard routine for prostate cancer diagnosis. Despite a variety of approaches exists to perform this gesture, transrectal sampling is the most widely used. This method consists in inserting a needle through a needle-guide fixed to an endorectal US probe in order to sample the carcinogenic tissue in the prostate. Generally, twelve biopsies are distributed in the prostate volume according to a systematic sextant scheme [1] (Fig.1). However, in some cases, additional targeted samples may be taken in a given area of interest corresponding to a $3 \mathrm{D}$ region earlier detected on an MRI image. The partial tissue information provided by the US images makes targeting precision hard. Moreover, pre-operative imaging does not provide information about the deformation and displacements occurring during the biopsy. Thereby, robotic and/or imageguided tools for assisted procedure are being developed $[2]$.

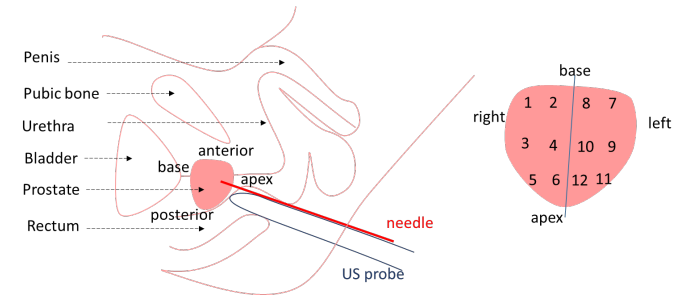

Fig. 1: Conventional sextant scheme.

Attaining a $2 \mathrm{~mm}$-targeting precision[3], can enable the delivery of localized treatments, with reduced collateral impact on the patient's life. In this paper we present the image-based control "AUTOFocus" for high-precision prostate samples targeting. A 6-DoFs robot is coupled to an imaging device for improved needle positioning.

\section{MATERIALS AND METHODS}

The robotized probe-holder: "Apollo" (Fig.2) is a 6-DoFs anthropomorphic robot [3], featuring 3 motorized joints and a back-drivable wrist, equipped with breaks for locking purposes. The robot, designed to assist prostate biopsies through co-manipulation, falls into the category of free-wrist robots. Apollo has 3 different modes:
- the FREE mode, characterized by high transparency and gravity compensation [3];

- the LOCKED mode to precisely hold the probe up to a target position [3];

- the AUTONOMOUS mode, to automatically displace the probe toward a desired anatomical location. This control mode is designed to target a desired position $T_{d}$ inside the prostate by computing the corresponding desired position of the robot wrist center, $W_{d}$. Tissues deformations (anus and rectum) are taken into account [2].

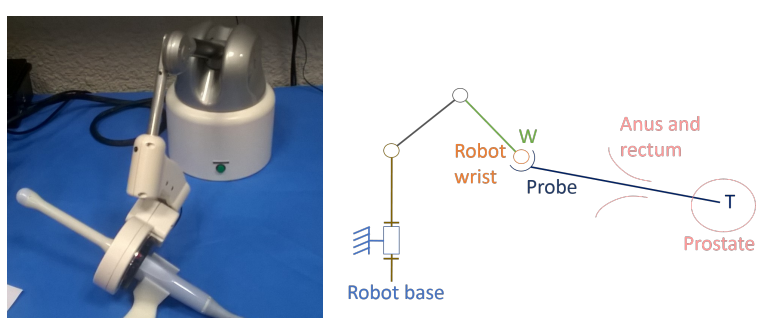

Fig. 2: Apollo robot V2 and kinematics scheme.

Imaging device: The Urostation ${ }^{\circledR}$, is a commercial device for elastic registration among 3D US images. It is used to measure the current needle and target positions: the urologist first records a set of $3 \mathrm{D}$ images to compute a prostate reference $3 \mathrm{D}$ volume. They can also define anatomical targets inside this reference. Throughout the examination, intraprocedure 3D images are used to update the current $3 \mathrm{D}$ volume with respect to the reference [4].

\section{AUTOFocus Mode:}

This mode enables to attain a higher precision for anatomical landmark-reaching gestures. As the current needle position can't be measured in real-time, conventional visual servoing control does not apply. However, positioning errors $\varepsilon_{T}$ between the desired target position $T_{d}$ and the current needle position $\mathrm{T}$ are estimated at each new $3 \mathrm{D}$ volume acquisition. Therefore, with an iterative process, the robot is able to precisely reach the cancer. The AUTOFocus mode is a semi-automatic closed loop process as it needs the surgeon's supervision to correctly converge toward the target in three steps: at each iteration the surgeon decides, based on the current error, whether to run another acquisition or to sample the tissue, as the precision is deemed satisfactory (step 4). The 


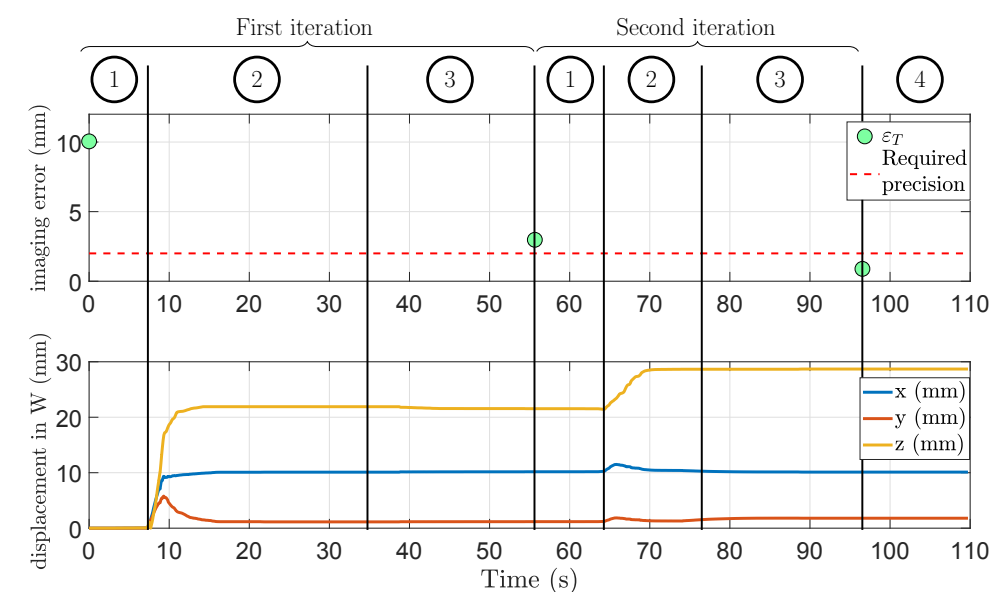

Fig. 3: The error computed by the AUTOFocus mode and the wrist robot displacements in two iterations.

first trial of the surgeon triggers the AUTOFocus mode, described in four steps (Fig.3):

$\rightarrow$ step 1: the surgeon's supervision;

- step 2: the robot displacement;

- step 3: the 3D-image acquisition and processing with the definition of a new $\varepsilon_{T}$;

- step 4: the precision is satisfactory and the surgeon can sample the prostate.

\section{RESULTS}

The experimental set-up is described on Fig.4. The probe tip is inserted through a phantom that reproduces both the mechanical and echogenecity of a prostate including the anus and the rectum deformations.

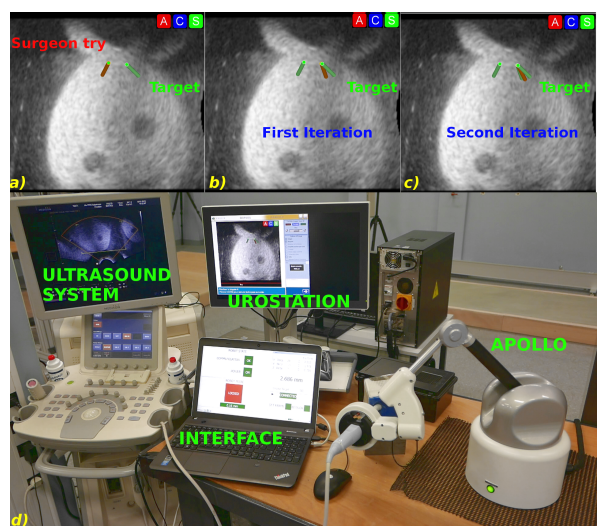

Fig. 4: Convergence to the target by small automatic movements a), b), c), experimental set-up d) and the convergence to the target in two iterations.

As aforementioned, the urologist first acquires the reference $3 \mathrm{D}$ volume of the prostate and specifies the target $\left(T_{d}\right)$. During the exploratory exam, the free mode is used. Then, 12 systematic biopsies are sampled and the robot locked mode helps the surgeon in stabilizing the gesture. If targeted biopsies are necessary, the surgeon manually displaces the probe toward the selected target. However, as the patient lies on the side, in a crouched position, a correct match- ing the US information with the actual prostate position impacts negatively on the targeting precision. The AUTOfocus mode is, then, able to minimize the residual errors by means of a new image acquisition. Fig. 3 and Fig.4 highlight the decrease of the pointing error, $\varepsilon_{T}$ : within two iterations, the error decreases from $10 \mathrm{~mm}$ down to below $2 \mathrm{~mm}$. In general, several iterations are needed to ensure convergence to the expected precision level: as we do not have access to the real time needle pose, any prostate deformation occurring in-between two consecutive iterations can't be accounted for; this might lead to unexpected variations of the error. However, the closed loop control ensure a precise and fast convergence (Fig.3).

\section{DISCUSSION}

A US-based, closed-loop control for precise probe displacement for prostate biopsies has been presented. Despite the high prostate deformability, a fast and satisfactory alignment of the needle axis with a biopsy target has been attained. Future work will include clinical studies to validate the advantages of robot-based platforms over the conventional routine.

\section{REFERENCES}

[1] A. Ouzzane, P. Coloby, JP. Mignard, JP. Allegre, M. Soulié, X. Rébillard, L. Salomon, A. Villers (2011) "Recommendations for best practice for prostate biopsy." Prog Urol 21(1):18.

[2] R. Chalard, D. Reversat, G.Morel, P.Mozer, MA. Vitrani (2018) "Precisely positioning the tip of an instrument inserted through an orifice with a free wrist robot: application to prostate biopsies," IJCARS.

[3] C. Poquet, P. Mozer, MA. Vitrani, and G. Morel (2015) "An endorectal ultrasound probe comanipulator with hybrid actuation combining brakes and motors," IEEE TMECH, pp.20(1)186-196.

[4] M. Baumann, P. Mozer, V. Daanen, and J. Troccaz (2012) "Prostate biopsy tracking with deformation estimation," Medical Image Analysis, pp.16(3)562576. 